\title{
Semi-Analytical Minimum Time Solution for the Optimal Control of a Vehicle subject to Limited Acceleration
}

\author{
Enrico Bertolazzi ${ }^{\mathrm{a}}$, Marco Frego ${ }^{\mathrm{b}}$ \\ ${ }^{a}$ DII - Dipartimento di Ingegneria Industriale \\ ${ }^{b}$ DISI - Dipartimento di Ingegneria e Scienza dell'Informazione
}

\begin{abstract}
A semi-analytic solution of the minimum time optimal control problem of a car-like vehicle is herein presented. The vehicle is subject to the effect of laminar (linear) and aerodynamic (quadratic) drag, taking into account the asymmetric bounded longitudinal accelerations. This yields a nonlinear differential equation of Riccati kind. The associated analytic solution exists in closed form, but is numerically ill conditioned in some situations, hence it is reformulated using asymptotic expansions that keep the numerical computations accurate and robust in all cases.

Therefore the proposed algorithm is designed to be fast and robust in sight to be the fundamental module of a more extended optimal control problem that considers a given clothoid curve as the trajectory and the presence of a constraint on the lateral acceleration of the vehicle. The numerical stability of the computation for limit values of the parameters is essential as showed in the numerical tests.
\end{abstract}

Keywords: Nonlinear Dynamic, Optimal Control, Semi-analytic solution, Asymptotic Expansion, Riccati ODE, Clothoid.

\section{Introduction and Motivation}

An important objective in the field of mobile robotics since the first papers of Dubins [16] is to find the optimal trajectory between two points with specified tangents vectors and velocities. Moreover, other non dynamic constraints $[6,22,34]$ are considered, such as the geometric continuity of the path $[33,38]$, the restrictions on the maximum curvature $[23,35]$, the avoidance of obstacles $[3,7,20]$. There are mainly two global views of this problem: the first tries to solve in one shot the generation of the path and the optimal law to travel it, the second splits the problem into two separate parts: path generation and optimisation of the dynamics. The first approach is done essentially numerically, considering very complex vehicle models, geometric constraints, and various other characteristics which approximate the real world in a very satisfactory way. The numeric solution obtained in this way is a formidable task by itself and it requires a deep knowledge of the physical problem and an efficient optimal control solver $[6,12,21,31,40]$. These solvers transform the problem into a Nonlinear Programming Problem (NLP) or into a Boundary Value Problem (BVP) that is efficiently solved by numerical methods [4, 24, 26, 27, 29, 39].

It is not possible to solve those problems analytically (and thus precisely and quickly) because they are too complex or simply they do not possess a closed form solution. Even when the analytic or semi-analytic solution exists [36, 37], it can be numerically ill conditioned or of impractical use, as in the present case. The purely numerical solutions are in general very good and accurate, but at the price of a very high computational cost, which implies that these methods are not suitable for real time computation. Thus existing numerical solver cannot be used in online simulations, where a quick feedback, e.g. in the case of an unexpected situation or a sudden event, must be managed.

On the other hand, splitting the problem in two sub-problems allows us to consider them separately, with the reasonable expectation that they become easier to tackle and admit an analytical solution. This is not usually the case, unless the problem is simplified via assumptions on the hypotheses and via linearisation of the differential equations of the

Email address: enrico.bertolazzi@unitn.it (Enrico Bertolazzi) 
dynamics. The advantage of such an approximate but analytic solution is that it is of quick evaluation and therefore suitable for real time applications. Complete analytic solutions are seldom obtained and almost only on toy problems of limited application, however, semi-analytical solutions are often a good trade-off between low computational times and usefulness.

With this second framework in mind, in [17] we solved the problem of a car-like vehicle travelling on a given clothoid, with assigned initial and final position, tangent and velocity. This results in an optimal control problem with a Riccati ODE in the dynamical system. The curve is traversed in minimum time, controlling the limited longitudinal acceleration and considering the bound on the maximum lateral acceleration of the vehicle, a quantity that connects the curvature of the path with the velocity of the vehicle, [17]. The complete problem is made up of several blocks: the algorithm for solving the $G^{1}$ Hermite Interpolation Problem with a clothoid [8], necessary to compute the path; the description of the car-like model and its simplification in order to find analytical useful solutions [17], the development of a fast and reliable tool that solves the ODEs of the basic optimal control (which is the aim of the present work), and a solution method for the optimal control problem with the constraint on the lateral acceleration. All these blocks are used inside a high level optimiser that constructs the trajectory of the car-like vehicle along a sequence of given points [18, 19, 34].

In this work, we focus on the fast and accurate numerical computation of the analytic solution of an optimal control problem arising from a Riccati dynamical system. We remark, since the limit of the lateral acceleration is a function of the state only (and not of the control variable), that we can solve the complete problem in two phases. In the first phase, we solve the OCP accounting for the longitudinal constraint only. The problem turns out to be classically Bang-Bang, yielding a switched hybrid system. We obtain analytic expressions for the optimal state variables as well as robust numerical routines to evaluate them in all cases, e.g., when the input parameters rise computational instabilities. Moreover, the various combinations of possible initial and final conditions produce many different analytic solution that are worth being studied independently because it is possible to synthesize them with only two stable expressions, even when the parameters of the problem lead to numerical instabilities. In the second phase, which is not scope of this work, we combine the obtained solutions together with the bound on the lateral acceleration in order to synthesize the optimal control as it is sketched in [17].

In Section 2 we present the Optimal Control Problem (OCP) with the description of the Riccati differential equation formulated with the time as the independent variable, we show the Bang-Bang nature of the solution and how to find the single switching point and the final total time of the maneuver, assuming that the analytic solutions of the ODEs of problem are available. The derivation of such analytic solutions is devoted to Section 3, where we give also the definition domains of the velocty $v(t)$ and space $s(t)$. The aim of Section 4 is to provide numerically stable versions of the solutions previously obtained. In Section 5 we introduce the change of variable from time $t$ to space $s$ for the velocity. In Section 6 we reformulate and solve the OCP presented in Section 2 but reformulated with the space as independent variable. Its solution is one nonlinear equation that we solve with the application of Newton's method. Section 7 shows the practical implementation of the stable equations presented in four different test cases for different variations of the parameters. The last section are the conclusions with some insights in application of the present work as a building block for more complex computations and OCP problems.

\section{The Optimal Control Problem}

The minimum time optimal control problem of a car-like vehicle that follows any smooth path, neglecting for the moment any constraints on the lateral acceleration, is [17]:

Find $a(t) \in\left[-\mathrm{a}_{\text {brake }}, \mathrm{a}_{\text {push }}\right]$ that minimizes the total time $T$ subject to:

$$
\begin{aligned}
& s^{\prime}(t)=v(t), \\
& v^{\prime}(t)=\mathrm{a}(t)-c_{0} v(t)-c_{1} v^{2}(t), \quad-\mathrm{a}_{\text {brake }} \leq a(t) \leq \mathrm{a}_{\text {push }}, \\
& s(0)=0, \quad s(T)=L, \quad v(0)=v_{i}, \quad v(T)=v_{f},
\end{aligned}
$$

where $T$ is the final time to be optimised, $s(t)$ represents the space variable in curvilinear coordinates, $v(t)$ is the velocity of the vehicle, $\mathrm{a}(t)$ is the controlled acceleration asymmetrically bounded for positive $\mathrm{a}_{\text {brake }}$ and $\mathrm{a}_{\text {push }}$, the laminar 
friction is given by the nonnegative coefficient $c_{0}$, the aerodynamic drag is modelled by the nonnegative coefficient $c_{1}$. All the boundary conditions are fixed: without loss of generality the initial position is 0 , the final position is $L$ (the length of the curve), the initial and final velocities are $v_{i}$ and $v_{f}$, respectively, both assumed nonnegative.

The solution of the OCP can be obtained via general optimal control software, as described [6, 9, 10, 11, 13, 21, 31], where various methods for OCP are presented. However they cannot take advantage of the analytic solution and hence, although being more general purpose, they are slower than the present method when applied to this specific problem.

We proceed by showing the nature of the optimal control using the Maximum Principle of Pontryagin. The Hamiltonian of the minimum time optimal control problem (1) is

$$
\mathcal{H}\left(s, v, \lambda_{1}, \lambda_{2}, \mathrm{a}\right)=1+\lambda_{1} v+\lambda_{2}\left(\mathrm{a}-c_{0} v-c_{1} v^{2}\right)
$$

and the control a appears linearly. Its optimal synthesis is obtained from Pontryagin's Maximum (minimum) Principle (PMP), and is

$$
\mathrm{a}(t)=\underset{\mathrm{a} \in\left[-\mathrm{a}_{\text {brake }}, \mathrm{a}_{\text {push }}\right]}{\arg \min } \mathcal{H}\left(s(t), v(t), \lambda_{1}(t), \lambda_{2}(t), \mathrm{a}\right)= \begin{cases}\mathrm{a}_{\text {push }} & \text { if } \lambda_{2}(t)<0, \\ -\mathrm{a}_{\text {brake }} & \text { if } \lambda_{2}(t)>0, \\ \mathrm{a}_{\text {sing }} & \text { if } \lambda_{2}(t)=0\end{cases}
$$

Hence the solution of the PMP produces a typical Bang-Bang controller. The term $\mathrm{a}_{\text {sing }}$ represents a possible singular control when $\lambda_{2}$ is identically zero on an interval. The equations for the adjoint variables are

$$
\lambda_{1}^{\prime}=0, \quad \lambda_{2}^{\prime}=\lambda_{1}\left(c_{0}+2 c_{1} v\right)-\lambda_{1} .
$$

As the state variables have complete boundary conditions, no initial or final conditions are required for the two multipliers. The singular control $a_{\text {sing }}$ is derived by using the above expressions for the multipliers, but according to a well known result in Optimal Control Theory [5], the solution of problem (1), if it exists, has at most one switching instant (denoted as $t_{\sigma}$ ) when the control value changes.

Lemma 1. The optimal control for problem (1), if exists, has at most one switching point and the control law is Bang-Bang.

Proof. See [5]. The idea is that the multiplier $\lambda_{2}$, in absence of bounds on the state variables, is strictly monotone increasing, hence there is at most one isolated zero and no singular arcs can exist.

This implies that the optimal control has to be chosen from a family of four candidate controls. The first and second correspond to pure acceleration (i.e., $\mathrm{a}(t) \equiv \mathrm{a}_{\text {push }}$ ) or braking manoeuvre (i.e., $\mathrm{a}(t) \equiv-\mathrm{a}_{\text {brake }}$ ), the third and fourth are a combination of the two. Having explicit expressions for the optimal states, with the complete boundary conditions, the solution of each case is obtained by the solution of a nonlinear system in two unknowns: the switching time $t_{\sigma}$ and the final time $T$. It is possible to rule out the case of a braking manoeuvre followed by an acceleration because it is not optimal. Moreover, the cases of pure acceleration or braking are very unlikely, because the boundary conditions should exactly satisfy the boundary value problem (1). Hence, only the case of acceleration and braking with a switching time (possibly degenerate zero length interval at the extrema of the time interval) is herein considered. It follows that the solution is given by the intersection at the switching point $t_{\sigma}$ of the two curves of the velocity and the space $v(t)$ and $s(t)$. The closed form solution of $v(t)$ and $s(t)$ and its accurate computation is a difficult and important part, therefore it is postponed to Section 3.

We are here interested to find the existence conditions for the optimal control of problem (1). They must depend on the assigned initial and final positions and velocities. We have to ensure that there is enough acceleration (respectively deceleration) together with the initial and final velocities to travel the curve. Those values should be compatible with the extremal manoeuvres of pure acceleration and pure braking.

If the final velocity $v_{f}$ is greater than the reference velocity $v_{f}^{\max }$ obtained starting from $v_{i}$ with maximum acceleration, then the problem does not have solution. Reference velocity $v_{f}^{\min }$ is the final velocity obtained starting from $v_{i}$ 
with maximum deceleration and $v_{f}^{\min }=0$ if the maximum deceleration produces a solution with $v(t)=0$ for $s(t)<L$, i.e., the vehicle is stopped before to reach the final distance $L$. If the final velocity $v_{f}$ is lower than the reference velocity $v_{f}^{\min }$ the problem is not solvable.

In the other cases a solution exists. Those limits values are discussed in the next sections, together with the analytic expression of the integration of the differential equations.

The complete solution of the Optimal Control Problem 1 is reduced to finding the optimal switching instant $t_{\sigma}$ and the final minimum time $T$. This is done equating the arcs of positive acceleration of velocity and space with the corresponding arcs of negative acceleration. It results a system of two nonlinear equations in the unknowns $t_{\sigma}$ and $T$ :

$$
v_{L}\left(t_{\sigma}\right)=v_{R}\left(t_{\sigma}-T\right), \quad s_{L}\left(t_{\sigma}\right)=s_{R}\left(t_{\sigma}-T\right) .
$$

Where $s_{L}(t)$ and $v_{L}(t)$ are the solutions of the ODE

$$
\begin{cases}s^{\prime}(t)=v(t), & s(0)=0 \\ v^{\prime}(t)=\mathrm{a}_{\text {push }}-c_{0} v(t)-c_{1} v^{2}(t), & v(0)=v_{i}\end{cases}
$$

whereas $s_{R}(t)$ and $v_{R}(t)$ are the solutions of the ODE

$$
\begin{cases}s^{\prime}(t)=v(t), & s(T)=L \\ v^{\prime}(t)=-\mathrm{a}_{\text {brake }}-c_{0} v(t)-c_{1} v^{2}(t), & v(T)=v_{f}\end{cases}
$$

The next parts of the present work are devoted to the computation of the analytic expressions of $s(t), v(t)$ and to the study their numeric stable implementation. The nonlinear system (2) can be further reduced to the computation of the zero of a single nonlinear equation. From this reduction it is possible to show that there is a unique solution and that the Newton method always converges. However this reduction is possible at the price of an involved change of variable, hence we prefer to solve directly system (2) which is well behaved with a small computational cost.

\section{Formal Analytic Solution of Riccati ODE}

From the analysis of the optimal control problem in the previous section, we conclude that the second differential equation of (1), i.e.,

$$
v^{\prime}(t)=\mathrm{a}(t)-c_{0} v(t)-c_{1} v(t)^{2}, \quad v(0)=v_{\circ} \geq 0,
$$

should be considered only for piecewise constant values of the control a $(t)$. This simplifies a lot the integration of the ODE, which is a differential equation of Riccati that can be solved only for a small class of functions a $(t)$, $[1,30,41]$. The constant functions are in this class and allow to solve the ODE by means of separation of variables or transforming the Riccati into a Bernoulli and then with standard techniques into a linear ODE. The shape of the solution varies according to the value of the initial condition, see Figure 1 for a graphic reference.

For constant positive accelerations a $>0$ and positive $c_{1}>0$, the threshold is given by the asymptotic value $v_{\infty}$. For initial conditions $v_{\circ}<v_{\infty}$, the velocity is monotone increasing towards the asymptote $v_{\infty}$, for $v_{\circ}>v_{\infty}$ the velocity is monotone decreasing towards the asymptotic value $v_{\infty}$, lastly, if $v_{\circ}=v_{\infty}$ the velocity is constant.

For negative accelerations a $<0$, the situation is slightly different, and the sign of the quantity $c_{0}^{2}+4 \mathrm{a} c_{1}$ must be considered: this leads to two cases. Also the cases of $\mathrm{a}=0, c_{0} \approx 0$ and/or $c_{1} \approx 0$ should be analysed, because they originate numerical difficulties when evaluating the analytic expression of $v(t)$. Once the explicit expression for $v(t)$ is given, the integration of the ODE for the space variable $s^{\prime}=v$ is possible in closed form. We have collected the various events in different cases, that we will discuss next.

A straightforward integration of (3) with a general initial condition $v_{\circ}$ yields a long expression, which involves inverse trigonometric functions and their hyperbolic analogue according to the sign of the acceleration, the integration of the velocity has those functions nested inside a logarithm, making $v(t)$ and $s(t)$ difficult to accurately evaluate. Thus, in 


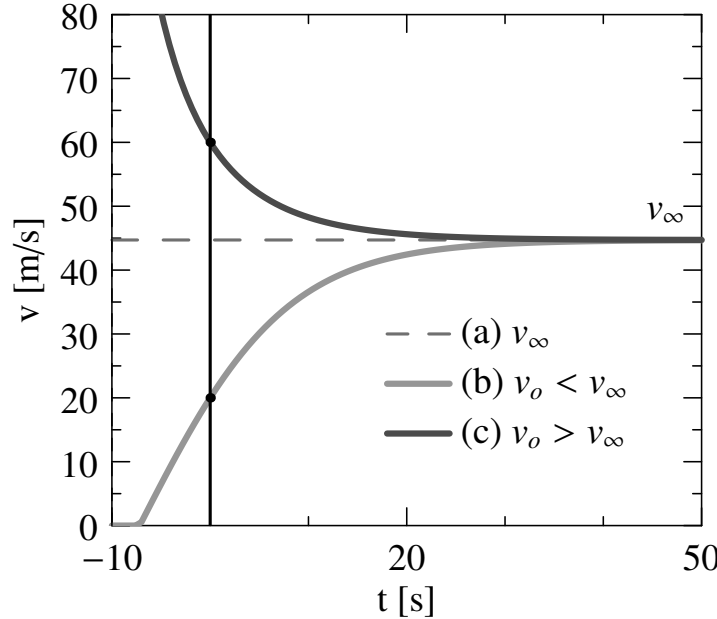

1.1 Velocity plot for $\mathrm{a} \geq 0$. Cases (a), (b) and (c). Case (a) is the horizontal line of constant velocity $v(t)=v_{\infty}$. The lower curve represents case (b), when $v_{\circ}$ is lower than the asymptotic velocity $v_{\infty}$; case (c) is the upper curve, when $v_{\circ}$ is higher than $v_{\infty}$.

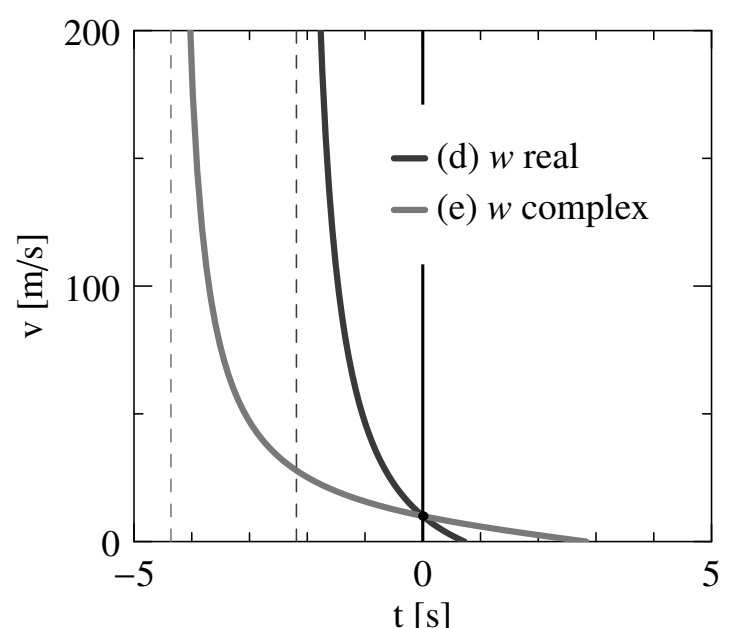

1.2 Velocity plot for a $<0$. Cases (d) and (e). Arcs indicate negative constant acceleration $\mathrm{a}=-\mathrm{a}_{\text {brake. There are two cases }}$ according to $w$. When $w$ is real, we fall in case (d), when $w$ is complex we fall in case (e).

Figure 1: The five cases of velocity and travelled space as listed in Lemma 3. In all cases, the acceleration is constant and positive. The green arcs indicate positive constant control $\mathrm{a}=\mathrm{a}_{\mathrm{push}}$, red arcs have negative constant acceleration $\mathrm{a}=-\mathrm{a}_{\text {brake }}$.

some of the cases presented next, the solution of equation (3) is manipulated in order to use a more compact and stable formula with some auxiliary constants that are here introduced:

$$
\begin{array}{llll}
w:=\sqrt{c_{0}^{2}+4 \mathrm{a} c_{1}}, & \alpha:=\frac{w+c_{0}}{2}, & \beta & :=\frac{w-c_{0}}{2}, \\
\gamma:=c_{1} v_{\circ}+\alpha, & v_{\infty}:=\frac{\beta}{c_{1}}=\frac{\mathrm{a}}{\alpha}, & \mathrm{a}_{0}:=\left(c_{1} v_{\circ}+c_{0}\right) v_{\circ} \\
\theta:=\arctan \frac{|w|}{c_{0}}, & \theta_{0}:=\arctan \frac{v_{\circ}|w|}{v_{\circ} c_{0}+2|\mathrm{a}|}, & \theta_{1}:=\arctan \frac{|w|}{2 c_{1} v_{\circ}+c_{0}}
\end{array}
$$

Notice that according to the values of $c_{0}, c_{1}$ and a, the value $w$ can be real (w.l.o.g. assumed positive) or complex and $\theta_{1}=\theta-\theta_{0}$. The constants in the first two lines of (4) are used to simplify expressions when $w$ is real, the last line is used in the cases of $w$ complex. We are now able to state the main result of this section.

Lemma 2. The analytic solution of (3) with initial velocity $v_{\circ}$ takes the following forms:

$$
v(t)=v_{\circ}+\frac{\left(\mathrm{a}_{0}-\mathrm{a}\right) \mathcal{E}(t, w)}{1-\gamma \mathcal{E}(t, w)} w \geq 0 ; \quad v(t)=\frac{\sin \left(\theta_{0}-\frac{1}{2} t|w|\right)}{\sin \left(\theta_{1}+\frac{1}{2} t|w|\right)} \frac{\sqrt{|\mathrm{a}|}}{\sqrt{c_{1}}}, w \in \mathbb{C} \backslash \mathbb{R}
$$

where $\mathcal{E}(t, w):=\left(1-\mathrm{e}^{w t}\right) / w$ and $\theta, \theta_{0}$ and $\theta_{1}$ are defined in (4). The solution $v(t)$ is meaniningful only for finite nonnegative values.

Proof. It is straightforward to check that (5) satisfies the Riccati ODE (3).

As remarked before, the sign of the argument of the square root in $w$ is fundamental for determining the shape of the solution of the ODE. With this notation, the asymptotic velocity, when there is one, can be written for $a=a_{\text {push }}$ as $v_{\infty}=\mathrm{a}_{\text {push }} / \alpha \geq 0$.

The following lemma states the interval where the solution is defined. 
Table 1: Interval of existence and where the solution of (3) is finite and nonnegative

\begin{tabular}{|cl|ll|c|}
\hline case & condition & $\left(t_{\min }, t_{\max }\right)$ & $\left(s_{\min }, s_{\max }\right)$ & $v^{\prime}(t)$ \\
\hline (a) & $w \geq 0$ and $v_{\circ}=v_{\infty}$ & $(-\infty, \infty)$ & $(-\infty, \infty)$ & $=0$ \\
(b) & $w \geq 0$ and $v_{\circ}<v_{\infty}$ & {$\left[t_{\circ}, \infty\right)$} & {$\left[s_{\circ}, \infty\right)$} & $>0$ \\
(c) & $w \geq 0$ and $v_{\circ}>v_{\infty}$ & $\left(t_{\infty}, \infty\right)$ & $(-\infty, \infty)$ & $<0$ \\
(d) & $w \geq 0$ and $\mathrm{a}<0$ & $\left(t_{\infty}, t_{\circ}\right]$ & $\left(-\infty, s_{\circ}\right]$ & $<0$ \\
(e) & $w$ complex & $\left(t_{v_{\infty}}, t_{v_{\circ}}\right]$ & $\left(-\infty, s_{v_{\circ}}\right]$ & $<0$ \\
\hline
\end{tabular}

Lemma 3. The solution $v(t)$ of the problem (3) is finite and nonnegative for $t$ in the interval summarised in Table 1 and plotted in Figure 1. The values of $t_{\circ}, t_{\infty}, t_{v_{\circ}}$ and $t_{v_{\infty}}$ are the following:

$$
\begin{array}{rlrl}
t_{\circ}=\frac{1}{w} \log \left(1-w \frac{v_{\circ}}{\mathrm{a}+v_{\circ} \beta}\right), & & v_{\circ}<v_{\infty} \text { or } \mathrm{a}<0 \\
t_{\infty}=\frac{1}{w} \log \left(1-\frac{w}{\gamma}\right) & & v_{\circ}>v_{\infty} \text { or } \mathrm{a}<0 \\
t_{v_{\circ}}=\frac{2 \theta_{0}}{|w|}, & t_{v_{\infty}}=-\frac{2 \theta_{1}}{|w|}, & & w \text { complex }
\end{array}
$$

the value of $\theta_{0}$ and $\theta_{1}$ are given in (4).

Proof. We have 5 cases (summarized in Table 1) that are function of the parameters a, $v_{\circ}, c_{0}$ and $c_{1}$ :

(case a) This case corresponds to have $\alpha v_{\circ}=\mathrm{a}$, the constant solution, i.e. when the r.h.s. of ODE (3) vanishes, i.e. $\mathrm{a}=c_{0} v_{\circ}+c_{1} v_{\circ}^{2}$. Its nonnegative solution is $v_{\circ}=\mathrm{a} / \alpha$;

(case b) This case happens for $\alpha v_{\circ}<$ a when the numerator of (5) is zero for some $t$. A simple computation shows that the numerator has a single real root for $\alpha v_{\circ}<$ a or a $<0$. For $\alpha v_{\circ}<$ a the value of the root is $t_{\circ}$ of equation (6).

(case c) This case happens for $\alpha v_{\circ}>\mathrm{a} \geq 0$ when the denominator of (5) is zero for some $t$. A simple computation shows that the denominator has a single real root only if a $\left\langle\alpha v_{\circ}\right.$ and the value of the root is $t_{\infty}$ of equation (6).

(case d) This case happens when the denominator of (5) is zero for some $t_{\infty}$ and the numerator of (5) is zero for some $t_{\circ}$. This can happen only when a $<0$.

(case e) Equation (5) has denominator zero for some $t_{v_{\infty}}$ and numerator zero for some $t_{v_{\circ}}$. This zeros are the border of the interval.

The remaining part of the proof is a simple computation of the zeros of numerators and denominators.

The velocity has to be positive, but this is true on some intervals only. Table 1 summarises the intervals of nonnegative velocity in terms of time and also of travelled space. We focus now on the other state variable, the space $s$. The integration of (5) permits to define the travelled space and the explicit expression is contained in the next Lemma.

Lemma 4. Let $s(t)=\int_{0}^{t} v(\zeta) \mathrm{d} \zeta$ be the space travelled with velocity $v(t)$, then

$$
s(t)= \begin{cases}v_{\infty} t+c_{1}^{-1} \log \left(1-c_{1}\left(v_{\infty}-v_{\circ}\right) \mathcal{E}(-t, w)\right), & \text { for } w \geq 0 \\ c_{1}^{-1}\left(\log \left(\frac{\sin \left(\theta_{1}+\frac{1}{2} t|w|\right)}{\sin \theta_{1}}\right)-\frac{c_{0}}{2} t\right), & \text { for } w \text { complex }\end{cases}
$$

the value of $\theta_{0}$ and $\theta_{1}$ are defined in equation (4). 
Proof. As with Lemma 3, the expressions for $s(t)$ are obtained by standard integration techniques, it is an exercise to check that they satisfy $s^{\prime}(t)=v(t)$. Integrating (5) and noticing that $\alpha \beta=\mathrm{a} c_{1}$ :

$$
\begin{aligned}
s(t) & =\frac{\alpha v_{\circ}-\mathrm{a}}{\beta-c_{1} v_{\circ}} t+\frac{\mathrm{a}_{0}-\mathrm{a}}{\gamma(\gamma-w)} \log (1-\gamma \mathcal{E}(t, w)) \\
& =\frac{\alpha v_{\circ}-\mathrm{a}}{\beta-c_{1} v_{\circ}} \frac{\alpha+c_{1} v_{\circ}}{\alpha+c_{1} v_{\circ}} t+c_{1}^{-1} \log \left(1-\left(c_{1} v_{\circ}+\alpha\right) \mathcal{E}(t, w)\right) \\
& =-c_{1}^{-1} \alpha t+c_{1}^{-1} \log \left(1-\left(c_{1} v_{\circ}+\alpha\right) \mathcal{E}(t, w)\right) .
\end{aligned}
$$

Then using the equality $v_{\infty} c_{1}=\beta$, we have

$$
\begin{aligned}
s(t) & =v_{\infty} t-c_{1}^{-1}(\alpha+\beta) t+c_{1}^{-1} \log \left(1-\left(c_{1} v_{\circ}+\alpha\right) \mathcal{E}(t, w)\right) \\
& =v_{\infty} t+c_{1}^{-1} \log \left(\mathrm{e}^{-t w}-\left(c_{1} v_{\circ}+\alpha\right) \mathrm{e}^{-t w} \mathcal{E}(t, w)\right) \\
& =v_{\infty} t+c_{1}^{-1} \log \left(1-w \mathcal{E}(-t, w)+\left(c_{1} v_{\circ}+\alpha\right) \mathcal{E}(-t, w)\right) \\
& =v_{\infty} t+c_{1}^{-1} \log \left(1+\left(c_{1} v_{\circ}-\beta\right) \mathcal{E}(-t, w)\right) \\
& =v_{\infty} t+c_{1}^{-1} \log \left(1+\left(c_{1} v_{\circ}-c_{1} v_{\infty}\right) \mathcal{E}(-t, w)\right) .
\end{aligned}
$$

Now from the next equality, for constant $a, b, c$ and $d$,

$$
\frac{\mathrm{d}}{\mathrm{d} t} \frac{(c t-a) \cos (a+b)-\log (\sin (c t+b)) \sin (a+b)}{c}=\frac{\sin (c t-a)}{\sin (c t+b)}
$$

and by posing $a=\theta_{0}, b=\theta_{1}, c=|w| / 2$ with the angle definitions of (4), we obtain (7).

As a corollary, it is possible to determine the special values $s_{\circ}$ and $s_{v_{0}}$.

Corollary 5. The values of $s_{\circ}$ and $s_{v_{\circ}}$ of Table 1 are:

$$
s_{\circ}=\frac{1}{w}\left[\frac{\beta}{c_{1}} \log \left(1-v_{\circ} \frac{c_{1}}{\beta}\right)+\frac{\alpha}{c_{1}} \log \left(1+v_{\circ} \frac{c_{1}}{\alpha}\right)\right], \quad s_{v_{\circ}}=\frac{1}{2 c_{1}}\left[\log \left(1+\frac{\mathrm{a}_{0}}{|\mathrm{a}|}\right)-c_{0} t_{v_{\circ}}\right],
$$

where $t_{v_{\circ}}$ is defined in (6).

Proof. We use the relations

$$
\mathcal{E}\left(-t_{\circ}, w\right)=\frac{1}{w}\left(1-\frac{\gamma v_{\infty}}{\gamma v_{\infty}-w v_{\circ}}\right)=\frac{v_{\circ}}{w v_{\circ}-\gamma v_{\infty}}=\frac{v_{\circ}}{w v_{\circ}-v_{\circ} \beta-\mathrm{a}}=\frac{v_{\circ}}{\alpha v_{\circ}-\alpha v_{\infty}}
$$

and (6) in (7), the two values $s\left(t_{\circ}\right)$ and $s\left(t_{v_{\circ}}\right)$ become respectively:

$$
\begin{aligned}
s\left(t_{\circ}\right) & =\frac{v_{\infty}}{w} \log \left(1-\frac{w}{\gamma} \frac{v_{\circ}}{v_{\infty}}\right)+\frac{1}{c_{1}} \log \left(1-c_{1}\left(v_{\infty}-v_{\circ}\right) \mathcal{E}\left(-t_{\circ}, w\right)\right) \\
& =\frac{v_{\infty}}{w} \log \left(1-\frac{w}{\gamma} \frac{v_{\circ}}{v_{\infty}}\right)+\frac{1}{c_{1}} \log \left(1+c_{1} \frac{v_{\circ}}{\alpha}\right) \\
& =\frac{\beta}{w c_{1}} \log \left(1-c_{1} \frac{w}{\gamma} \frac{v_{\circ}}{\beta}\right)+\frac{\alpha+\beta}{w c_{1}} \log \left(1+c_{1} \frac{v_{\circ}}{\alpha}\right) \\
& =\frac{\beta}{w c_{1}} \log \left(1-c_{1} \frac{w}{\gamma} \frac{v_{\circ}}{\beta}\right)+\frac{\beta}{w c_{1}} \log \left(1+c_{1} \frac{v_{\circ}}{\alpha}\right)+\frac{\alpha}{w c_{1}} \log \left(1+c_{1} \frac{v_{\circ}}{\alpha}\right) \\
& =\frac{1}{w}\left[\frac{\beta}{c_{1}} \log \left(1-\frac{c_{1}}{\beta} v_{\circ}\right)+\frac{\alpha}{c_{1}} \log \left(1+\frac{c_{1}}{\alpha} v_{\circ}\right)\right],
\end{aligned}
$$


and

$$
\begin{aligned}
s\left(t_{v_{\mathrm{o}}}\right)+\frac{c_{0}}{2 c_{1}} t_{v_{\mathrm{o}}} & =\frac{1}{c_{1}} \log \left(\frac{\sin \left(\theta-\theta_{0}+\frac{1}{2} t_{v_{\mathrm{o}}}|w|\right)}{\sin \left(\theta-\theta_{0}\right)}\right)=\frac{1}{c_{1}} \log \left(\frac{\sin \theta}{\sin \left(\theta-\theta_{0}\right)}\right) \\
& =-\frac{1}{c_{1}} \log \left(\frac{\sin \theta \cos \theta_{0}-\cos \theta \sin \theta_{0}}{\sin \theta}\right)=-\frac{1}{c_{1}} \log \left(\cos \theta_{0}-\frac{\sin \theta_{0}}{\tan \theta}\right) \\
& =-\frac{1}{c_{1}} \log \left(\cos \theta_{0}-\frac{c_{0}}{|w|} \sin \theta_{0}\right)=-\frac{1}{2 c_{1}} \log \left(\frac{|\mathrm{a}|}{\mathrm{a}_{0}+|\mathrm{a}|}\right) .
\end{aligned}
$$

Now bringing $\frac{c_{0}}{2 c_{1}} t_{v_{\mathrm{o}}}$ to the r.h.s. completes the proof.

Lemma 6. The acceleration $v^{\prime}(t)$ of Equation (3) is identically 0 or is of constant sign in the interval where $v(t) \geq 0$, the corresponding signs are collected in Table 1. Moreover, the space $s(t)$ is monotone increasing where $v(t) \geq 0$ and thus the inverse function $t(\hat{s})$, i.e. the solution of the problem $s\left(t, v_{0}\right)=\hat{s}$, is well defined.

Proof. From equation (3) with constant acceleration a, suppose that for a certain $t=\tau$ the velocity is $v^{\prime}(\tau)=0$. If we consider the shifted function $\tilde{v}^{\prime}(t)=v^{\prime}(t-\tau)$, we have $0=\mathrm{a}-\left(c_{0}-c_{1} \tilde{v}(0)\right) \tilde{v}(0)$ and from equation (5) of Lemma 2 the solution $\tilde{v}(t)$ is constant.

Corollary 7. The function $\tilde{v}(s)=v(t(s))$ satisfies the following ODE:

$$
\tilde{v}^{\prime}(s)=\frac{\mathrm{a}(s)}{\tilde{v}(s)}-c_{0}-c_{1} \tilde{v}(s), \quad \tilde{v}(0)=v_{\circ} \geq 0,
$$

that is, the ODE (3) in the new independent variable s. This change of variable will be used in next sections.

\section{Stable Computation of the Analytic Solutions}

Expressions (5) and (7) may be unstable or numerically inaccurate for $c_{0} \approx 0, c_{1} \approx 0$ or $w \approx 0$ or for $t \approx t_{\infty}$ or $t \approx t_{\mathrm{o}}$. Numerically accurate reformulation for expressions (5) and (7) are derived in this section. We define some auxiliary functions:

$$
\begin{aligned}
\mathcal{L}(t, c):=c^{-1} \log (1-c t), & \mathcal{E}(t, w):=w^{-1}\left(1-\mathrm{e}^{w t}\right), \\
\mathcal{S}(x, w):=w^{-1} \sin (w x), & \mathcal{A}(x, w):=w^{-1} \arctan (w x),
\end{aligned}
$$

and

$$
\begin{aligned}
\mathcal{F}\left(t, w, c_{0}\right) & :=\mathrm{e}^{t c_{0} / 2} \mathcal{G}\left(t, w, c_{0}\right), \\
\mathcal{G}\left(t, w, c_{0}\right) & :=\frac{1}{w}\left[\mathcal{E}\left(-t, \frac{w+c_{0}}{2}\right)+\mathcal{E}\left(t, \frac{w-c_{0}}{2}\right)\right],
\end{aligned}
$$

Lemma 8. The functions (9) and (10) are smooth in their definition domain and have the following converging Taylor 
expansions:

$$
\begin{aligned}
\mathcal{L}(t, c) & =-t \sum_{n=0}^{\infty} \frac{(c t)^{n}}{n+1}, & \mathcal{E}(t, w) & =-t \sum_{n=0}^{\infty} \frac{(w t)^{n}}{(n+1) !} \\
\mathcal{S}(x, w) & =x \sum_{n=0}^{\infty} \frac{\left(-\left(w x^{2}\right)\right)^{n}}{(2 n+1) !}, & \mathcal{A}(x, w) & =x \sum_{n=0}^{\infty} \frac{\left(-(w x)^{2}\right)^{n}}{2 n+1}, \\
\mathcal{F}\left(t, w, c_{0}\right) & =-\sum_{n=1}^{\infty} \frac{f_{n} t^{2 n}}{2^{2 n}(2 n) !}\left[4+\frac{2 c_{0} t}{2 n+1}\right], & f_{n} & =\frac{w^{2 n}-c_{0}^{2 n}}{w^{2}-c_{0}^{2}}, \\
\mathcal{G}\left(t, w, c_{0}\right) & =-\sum_{n=1}^{\infty} f_{n} \mathrm{e}^{h_{n}(t)}\left[4+\frac{2 c_{0} t}{2 n+1}\right], & h_{n}(t) & =\sum_{j=1}^{2 n} \log \frac{|t|}{2 j}-\frac{t c_{0}}{2},
\end{aligned}
$$

and the term $f_{n}$ is computed by the following recurrence:

$$
\text { Initial value: } \quad\left\{\begin{array} { l } 
{ g _ { 1 } = 1 , } \\
{ f _ { 1 } = 1 , }
\end{array} \quad \text { Recurrence: } \quad \left\{\begin{array}{l}
g_{n+1}=g_{n} c_{0}^{2}, \\
f_{n+1}=f_{n} w^{2}+g_{n+1} .
\end{array}\right.\right.
$$

Proof. The Taylor series for $\mathcal{L}, \mathcal{E}, \operatorname{sinc}^{1}, \mathcal{A}$ are straightforward. Some effort is necessary to derive the expansion for $\mathcal{F}\left(t, w, c_{0}\right)$ and $\mathcal{G}\left(t, w, c_{0}\right)$. To compute $(10)$, we multiply it by $\left(w^{2}-c_{0}^{2}\right) / 2$ so that we obtain:

$$
\begin{aligned}
\frac{w^{2}-c_{0}^{2}}{2} \mathcal{F}\left(t, w, c_{0}\right) & =2 \mathrm{e}^{t c_{0} / 2}-\mathrm{e}^{-t w / 2}-\mathrm{e}^{t w / 2}+\frac{c_{0}}{w}\left(\mathrm{e}^{-t w / 2}-\mathrm{e}^{t w / 2}\right) \\
& =\sum_{n=1}^{\infty} \frac{t^{n}}{2^{n} n !}\left(2 c_{0}^{n}-\left((-w)^{n}+w^{n}\right)+\frac{c_{0}}{w}\left((-w)^{n}-w^{n}\right)\right) \\
& =\sum_{n=3,5,7, \ldots}^{\infty} \frac{t^{n}\left(2 c_{0}^{n}-2 c_{0} w^{n-1}\right)}{2^{n} n !}+\sum_{n=2,4,5, \ldots}^{\infty} \frac{t^{n}\left(2 c_{0}^{n}-2 w^{n}\right)}{2^{n} n !} \\
& =\sum_{n=1}^{\infty} \frac{t^{2 n+1} c_{0}\left(c_{0}^{2 n}-w^{2 n}\right)}{2^{2 n}(2 n+1) !}+\sum_{n=1}^{\infty} \frac{t^{2 n}\left(c_{0}^{2 n}-w^{2 n}\right)}{2^{2 n-1}(2 n) !} \\
& =\sum_{n=1}^{\infty} \frac{t^{2 n}}{2^{2 n}(2 n) !}\left[2+\frac{c_{0} t}{2 n+1}\right]\left(c_{0}^{2 n}-w^{2 n}\right) .
\end{aligned}
$$

If we define the general term $f_{n}:=\left(w^{2 n}-c_{0}^{2 n}\right) /\left(w^{2}-c_{0}^{2}\right)$ then (11) is readily obtained. Those functions are smooth and numerically stable even when the arguments $w$ and $c_{0}$ approach zero. Notice that an efficient computation of the series (10) uses the recurrence for $f_{n}$. The use of the recurrence is mandatory to avoid cancellation errors or numerical overflows, hence the stable and efficient algorithm to evaluate (11) is summarised in the next equation:

$$
\mathcal{G}\left(t, w, c_{0}\right)=-\sum_{n=1}^{\infty} \mathrm{e}^{h_{n}(t)}\left[4+\frac{2 c_{0} t}{2 n+1}\right], \quad h_{n}(t)=2 n \log \frac{|t|}{2}-\frac{t c_{0}}{2}-\log ((2 n) !),
$$

where

$$
h_{n}(t)=2 n \log \frac{|t|}{2}-\frac{t c_{0}}{2}-\sum_{j=1}^{2 n} \log j=\sum_{j=1}^{2 n}\left(\log \frac{|t|}{2}-\log j\right)-\frac{t c_{0}}{2}=\sum_{j=1}^{2 n} \log \frac{|t|}{2 j}-\frac{t c_{0}}{2}
$$

\footnotetext{
${ }^{1}$ Notice that we use the unnormalised sinc function, defined, for $x \neq 0$ and extended for $x=0$ with its limit.
} 
and the remaining part follows easily.

The computation of the special values $t_{\circ}, t_{\infty}, t_{v_{\circ}}, v_{\infty}$ of Table 1 and corresponding values of $s(t, v)$ is critical and must be accurate, otherwise complex arguments or logarithms of negative values are very likely. Using Lemma 8 , the stable computation of the ranges in (6) is written in terms of the smooth functions introduced in (11):

$$
\begin{array}{rlrl}
t_{\circ} & =\mathcal{L}\left(\frac{v_{\circ}}{\mathrm{a}+v_{\circ} \beta}, w\right), & t_{\infty}=\mathcal{L}\left(\frac{1}{\gamma}, w\right), & w \geq 0 \\
t_{v_{\circ}}=2 \mathcal{A}\left(\frac{v_{\circ}}{v_{\circ} c_{0}+2|\mathrm{a}|},|w|\right), & t_{v_{\infty}}=t_{v_{\circ}}-2 \mathcal{A}\left(\frac{1}{c_{0}},|w|\right), & w \text { complex. }
\end{array}
$$

An algorithmic version of the computation of the necessary constants for the semi-analytic solution of the OCP (1) is given in Algorithm 1.

\subsection{Stable speed computation}

With the previously introduced formulas, the expressions of Lemma 2 are rewritten in the numerically stable version in the next Proposition.

Proposition 9. The speed $v(t)$ is numerically stable for parameters $w \approx 0$ or $t \approx t_{\circ}$ or $t \approx t_{\infty}$ if computed as follows (An algorithmic version for computing $v(t)$ is presented in Algorithm 2 ). For $w$ real, the velocity is rewritten as:

$$
v(t)=\frac{p(t)}{q(t)}+\left\{\begin{array} { l l } 
{ 0 } & { \text { if } | t - t _ { \circ } | \leq \epsilon , } \\
{ v _ { \circ } } & { \text { otherwise, } }
\end{array} \left\{\begin{array}{l}
p(t)= \begin{cases}\left(\alpha v_{\circ}-\mathrm{a}\right) \mathcal{E}\left(t-t_{\circ}, w\right) & \text { if }\left|t-t_{\circ}\right| \leq \epsilon, \\
\left(\mathrm{a}_{0}-\mathrm{a}\right) \mathcal{E}(t, w) & t>0, \\
\left(\mathrm{a}-\mathrm{a}_{0}\right) \mathcal{E}(-t, w) & t \leq 0,\end{cases} \\
q(t)= \begin{cases}(w-\gamma) \mathcal{E}\left(t-t_{\infty}, w\right) & \text { if }\left|t-t_{\infty}\right| \leq \epsilon, \\
1-\gamma \mathcal{E}(t, w) & t>0, \\
1+(\gamma-w) \mathcal{E}(-t, w) & t \leq 0 .\end{cases}
\end{array}\right.\right.
$$

When $w$ is complex, a stable computation is:

$$
v(t)=\frac{v_{\circ} \cos \left(\frac{1}{2} t|w|\right)-\left(c_{0} v_{\circ}+2|\mathrm{a}|\right) \mathcal{S}\left(\frac{1}{2} t,|w|\right)}{\cos \left(\frac{1}{2} t|w|\right)+\left(2 c_{1} v_{\circ}+c_{0}\right) \mathcal{S}\left(\frac{1}{2} t,|w|\right)}
$$

Proof. Case $w \geq 0$ real. Notice that from (5) it is possible to write for $v$,

$$
v_{\circ}+\frac{\left(\mathrm{a}_{0}-\mathrm{a}\right) \mathcal{E}(t, w)}{1-\gamma \mathcal{E}(t, w)}=\frac{v_{\circ}-\left(\mathrm{a}+v_{\circ} \beta\right) \mathcal{E}(t, w)}{1-\gamma \mathcal{E}(t, w)}=v_{\circ}+\frac{\left(\mathrm{a}-\mathrm{a}_{0}\right) \mathcal{E}(-t, w)}{1+(\gamma-w) \mathcal{E}(-t, w)},
$$

therefore for the numerator, we have:

$$
\begin{aligned}
v_{\circ}-\left(\mathrm{a}+v_{\circ} \beta\right) \mathcal{E}(t, w) & =v_{\circ}-\left(\mathrm{a}+v_{\circ} \beta\right) w^{-1}\left(\mathrm{e}^{-t_{\circ} w}-\mathrm{e}^{\left(t-t_{\circ}\right) w}\right) \mathrm{e}^{t_{\circ} w} \\
& =v_{\circ}-\left(\mathrm{a}+v_{\circ} \beta\right)\left(-\mathcal{E}\left(-t_{\circ}, w\right)+\mathcal{E}\left(t-t_{\circ}, w\right)\right) \mathrm{e}^{t_{\circ} w} \\
& =v_{\circ}-\left(\mathrm{a}+v_{\circ} \beta\right)\left(\mathcal{E}\left(t_{\circ}, w\right)+\mathcal{E}\left(t-t_{\circ}, w\right)\left(1-\frac{w}{\gamma} \frac{v_{\circ}}{v_{\infty}}\right)\right) \\
& =v_{\circ}-\left(\mathrm{a}+v_{\circ} \beta\right)\left(\frac{1}{\gamma} \frac{v_{\circ}}{v_{\infty}}+\mathcal{E}\left(t-t_{\circ}, w\right)\left(1-\frac{w}{\gamma} \frac{v_{\circ}}{v_{\infty}}\right)\right) \\
& =\mathcal{E}\left(t-t_{\circ}, w\right)\left(\mathrm{a}+v_{\circ} \beta-w v_{\circ}\right) \quad\left[\mathrm{a}+v_{\circ} \beta=\gamma v_{\infty}\right] \\
& =\mathcal{E}\left(t-t_{\circ}, w\right)\left(\mathrm{a}-\alpha v_{\circ}\right)
\end{aligned}
$$


And for the denominator,

$$
\begin{aligned}
1-\gamma \mathcal{E}(t, w) & =1-\gamma w^{-1}\left(\mathrm{e}^{-t_{\infty} w}-\mathrm{e}^{\left(t-t_{\infty}\right) w}\right) \mathrm{e}^{t_{\infty} w} \\
& =1-\gamma\left(-\mathcal{E}\left(-t_{\infty}, w\right)+\mathcal{E}\left(t-t_{\infty}, w\right)\right) \mathrm{e}^{t_{\infty} w} \\
& =1-\gamma\left(\mathcal{E}\left(t_{\infty}, w\right)+\mathcal{E}\left(t-t_{\infty}, w\right)\left(1-w \gamma^{-1}\right)\right) \\
& =1-\gamma\left(\gamma^{-1}+\mathcal{E}\left(t-t_{\infty}, w\right)\left(1-w \gamma^{-1}\right)\right) \\
& =\mathcal{E}\left(t-t_{\infty}, w\right)(w-\gamma) .
\end{aligned}
$$

Case $w$ complex. The standard expansion of the trigonometric functions in (5) yields immediately (13).

\subsection{Stable space computation}

We restate here the numerically stable formulas of Lemma 4.

Lemma 10. The space $s(t)$ of formula $(7)$ is numerically stable for parameters $w \approx 0$, or $t \approx t_{\circ}$ or $t \approx t_{\infty}$, if computed as follows. For $w$ real, $s(t)$ can be rewritten as:

$$
s(t)=v_{\infty} t+\mathcal{L}\left(\left(v_{\infty}-v_{\circ}\right) \mathcal{E}(-t, w), c_{1}\right)=\mathcal{L}\left(\mathrm{a} \mathcal{G}\left(t, w, c_{0}\right)-v_{\circ} \mathcal{E}(-t, w) \mathrm{e}^{\beta t}, c_{1}\right),
$$

where we use the second equation when $v_{\infty} \gg v_{\circ}$. For $w$ complex and $c_{1} \gg 0$ a stable computation is:

$$
s(t)=\frac{1}{c_{1}} \log \left(\left(2 c_{1} v_{\circ}+c_{0}\right) \mathcal{S}\left(\frac{t}{2},|w|\right)+\cos \frac{t|w|}{2}\right)-\frac{c_{0} t}{2 c_{1}},
$$

the case $c_{1} \approx 0$ is considered in the next lemma.

Proof. Case $w \geq 0$ real. In this case equation (5) becomes, after some manipulations:

$$
\begin{aligned}
s(t) & =v_{\infty} t+c_{1}^{-1} \log \left(1-c_{1}\left(v_{\infty}-v_{\circ}\right) \mathcal{E}(-t, w)\right) \\
& =c_{1}^{-1} \beta t+c_{1}^{-1} \log \left(1-c_{1}\left(v_{\infty}-v_{\circ}\right) \mathcal{E}(-t, w)\right), \\
& =c_{1}^{-1} \log \left(\mathrm{e}^{\beta t}-c_{1}\left(v_{\infty}-v_{\circ}\right) \mathcal{E}(-t, w) \mathrm{e}^{\beta t}\right) \\
& =c_{1}^{-1} \log \left(1-\beta \mathcal{E}(t, \beta)-c_{1}\left(v_{\infty}-v_{\circ}\right) \mathcal{E}(-t, w) \mathrm{e}^{\beta t}\right), \\
& =c_{1}^{-1} \log \left(1-c_{1} v_{\infty}\left(\mathcal{E}(t, \beta)+w^{-1}\left(\mathrm{e}^{\beta t}-\mathrm{e}^{-\alpha t}\right)\right)+c_{1} v_{\circ} \mathcal{E}(-t, w) \mathrm{e}^{\beta t}\right), \\
& =c_{1}^{-1} \log \left(1-c_{1} \mathrm{a}^{-1}(\mathcal{E}(t, \beta)+\mathcal{E}(-t, \alpha))+c_{1} v_{\circ} \mathcal{E}(-t, w) \mathrm{e}^{\beta t}\right) .
\end{aligned}
$$

Case $w$ complex. Follows easily using (7) and the angle definitions in (5).

Lemma 11. Computation of $s(t)$ when $w$ is complex and $c_{1} \approx 0$ :

$$
s(t)= \begin{cases}\left(\mathrm{a}_{0}+|\mathrm{a}|\right) t^{2} Q\left(t \ell_{1}, \cos \theta_{1}\right)+v_{\circ} t & \left|t \ell_{1}\right| \leq 0.001 \\ u s e(15) & \left|t \ell_{1}\right|>0.001\end{cases}
$$

where

$$
\ell_{1}:=\sqrt{c_{1}\left(\mathrm{a}_{0}+|\mathrm{a}|\right)}, \quad \cos \theta_{1}:=\frac{2 c_{1} v_{\circ}+c_{0}}{2 \ell_{1}}, \quad \sin \theta_{1}:=\frac{|w|}{2 \ell_{1}}
$$


and $Q(\tau, c)$ can be approximated (when $\left.t \ell_{1} \approx 0\right)$ with

$$
Q(\tau, c)=-\frac{1}{2}+\frac{c \tau}{3}-\frac{2 c^{2}+1}{12} \tau^{2}+\frac{c^{2}+2}{15} c \tau^{3}-\frac{2 c^{4}+11 c^{2}+2}{90} \tau^{4}+\frac{2 c^{4}+26 c^{2}+17}{315} c \tau^{5}-\varepsilon(\tau, c) .
$$

The remainder is $|\varepsilon(\tau, c)| \leq 10^{-18}$.

Proof. Using (7), the definitions of the constants (17) and the angles $c_{0} / 2=\ell_{1} \cos \theta_{1}-c_{1} v_{\circ}$ we have

$$
s(t)=\frac{\mathrm{a}_{0}+|\mathrm{a}|}{\ell_{1}^{2}}\left(\log \left(\frac{\sin \left(t \ell_{1} \sin \theta_{1}\right)}{\tan \theta_{1}}+\cos \left(t \ell_{1} \sin \theta_{1}\right)\right)-t \ell_{1} \cos \theta\right)+v_{\circ} t
$$

By posing $\tau=t \ell_{1}$ and expanding with Taylor around $\tau$ :

$$
Q(\tau, c)=\frac{1}{\tau^{2}} \log \left(\frac{c}{s} \sin (\tau s)+\cos (\tau s)\right)-\frac{c}{\tau}, \quad s=\sqrt{1-c^{2}}
$$

The remainder can be estimated with

$$
\epsilon(\tau, c)=\frac{4 c^{6}+114 c^{4}+180 c^{2}+17}{2520} \tau_{\star}^{6}, \quad-\frac{\pi}{2} \leq \tau \leq \tau_{\star} \leq 0 .
$$

From the interval of definition $|\tau| \leq 0.001$, we have that $|\epsilon(\tau, c)| \leq 0.125 \tau_{\star}^{6}$ for $|\tau| \leq 0.001$, thus the error satisfies $|\epsilon(\tau, c)| \leq 1.25 \times 10^{-19}$.

Remark 1. The stable computation of (8) in Corollary 5 for $w \approx 0$ can be done using (14) or (16) with (12). An algorithmic version of the stable computation of the function $s(t)$ is given in Algorithm 3.

\section{Velocity as a function of the space $s$}

It is useful in the applications of this work, to have the velocity expressed as a function of the space and not of the time. This is the case for example when we want to add to the pure Bang-Bang problem a limitation on the lateral acceleration or constrain the problem with the friction ellipse. Those constraints are tipically functions of the space (e.g. the curvature of the trajectory). From Corollary 7, the function $\tilde{v}(s)$ and its derivatives can be computed via $t(s)$ with the relation $\tilde{v}(s)=v(t(s))$. The point of the problem is to invert the monotone function $s(t)$, or, in other words, to solve the following equation:

$$
t(\zeta) \text { solution of the problem } s(t)=\zeta
$$

The following well-known theorem gives enough conditions for the global convergence of Newtons method.

Theorem 12. Let $f(x)$ be twice continuously differentiable on the closed finite interval $[a, b]$ and let the following conditions be satisfied:

1. $f(a) f(b)<0$;

2. $f^{\prime}(x) \neq 0$ for all $x \in[a, b]$

3. $f^{\prime \prime}(x)$ either $\geq 0$ or $\leq 0$ for all $x \in[a, b]$

4. At the endpoint $a, b$ both $|f(a)| /\left|f^{\prime}(a)\right|<b-a$ and $|f(b)| /\left|f^{\prime}(b)\right|<b-$ a are satisfied;

Then Newton's method converges to the unique solution in $[a, b]$ for any choice of the initial guess in $[a, b]$.

Proof. See reference [15], Theorem 3.2 of page 104.

Corollary 13. Let $f(x)$ be twice continuously differentiable on the closed finite interval $[a, b]$ with 
case (b)

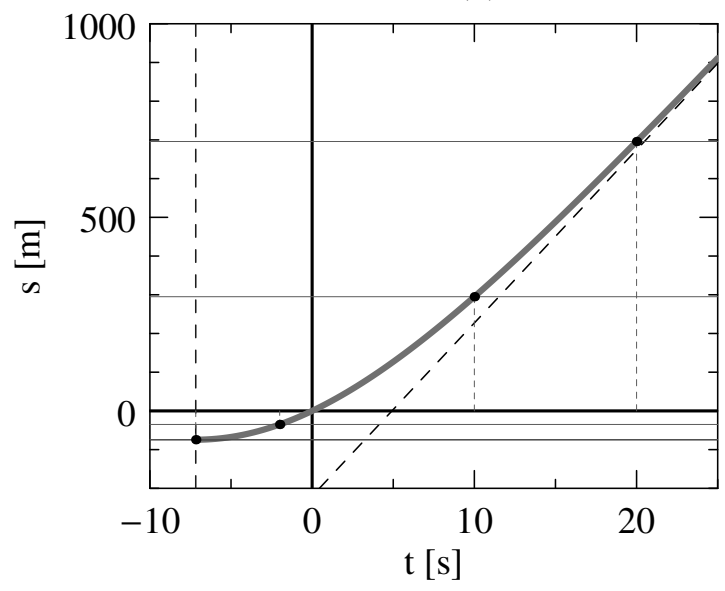

case (d)

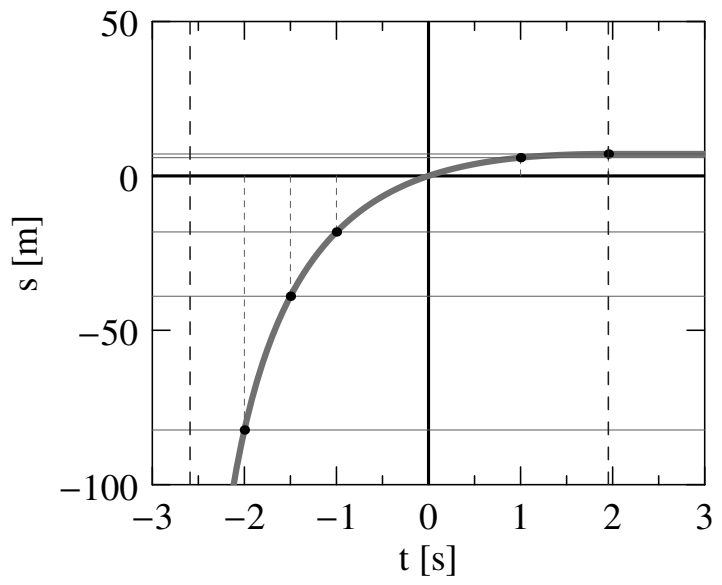

case (c)

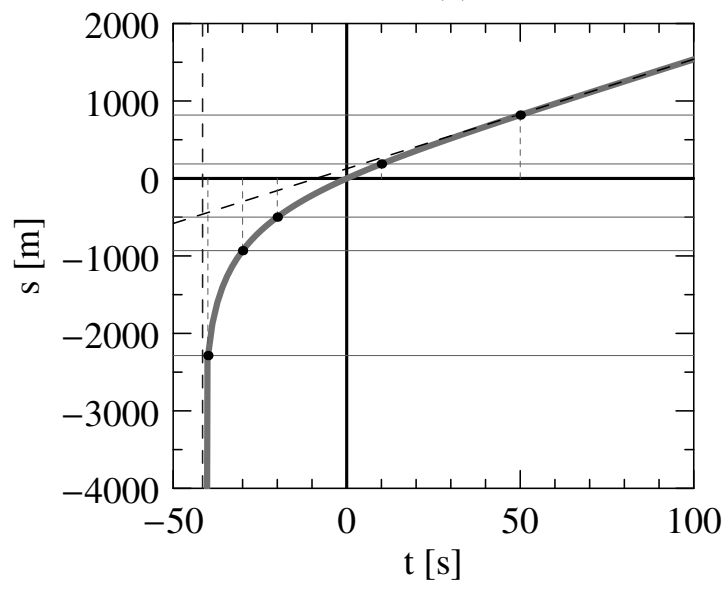

case (e)

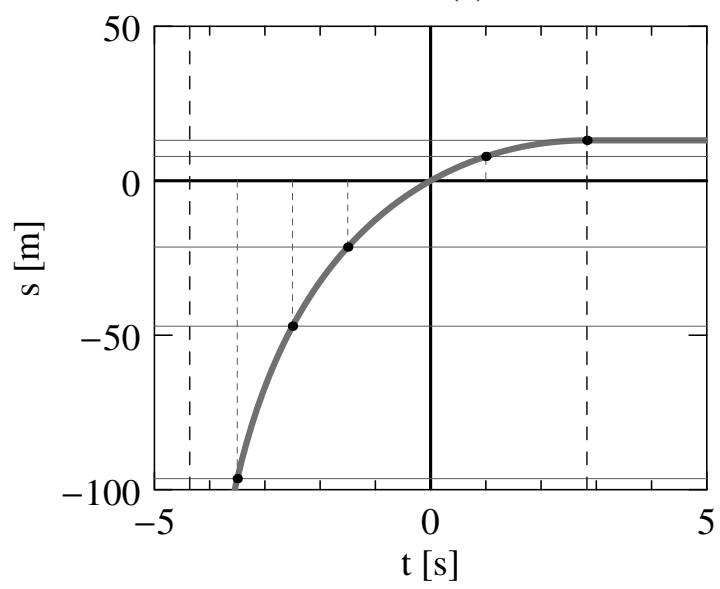

Figure 2: The five cases of the velocity and travelled space as listed in Lemma 3. In all those case the acceleration is constant and positive with constant control $\mathrm{a}=\mathrm{a}_{\text {push }}$.

1. $f(a) f(b)<0$;

2. $f^{\prime}(x) \neq 0$ for all $x \in[a, b]$

3. $f^{\prime \prime}(x) \geq 0$ for all $x \in[a, b]$

if $x_{0} \in[a, b]$ satisfies $f\left(x_{0}\right) \geq 0$ then the sequence generated by Newton's method converges monotonically to the unique solution in $[a, b]$. Analogously if $f^{\prime \prime}(x) \leq 0$ for all $x \in[a, b]$ and $f\left(x_{0}\right) \leq 0$.

Proof. Omitted

From problem (18) the evaluation of the inverse function $t(\zeta)$ is the solution of the problem $f(t)=0$ where $f(t)=s(t)-\zeta$. The function $f(t)$ has the following properties depending on the 5 cases (summarized in Table 1 ) that are function of the parameters $\mathrm{a}, v_{\circ}, c_{0}$ and $c_{1}$ :

case (a) $f(t)$ is linear and not constant;

case (b) up to case (e) $f^{\prime}(t) \neq 0$ and $f^{\prime \prime}(t) \geq 0$ or $f^{\prime \prime}(t) \leq 0$ for all $t \in\left(t_{\min }, t_{\max }\right)$. Moreover for $\epsilon$ small enough $f\left(t_{\min }+\epsilon\right) f\left(t_{\max }-\epsilon\right)<0$. 
Remark 2. The function $f(t)=s(t)-\zeta$ in all cases (a) up to (e) has a unique that satisfies $f(t)=0$. Moreover, Newton's method converges monotonically to the solution provided that initial guess $t_{\text {guess }}$ is chosen accordingly to Corollary 13.

A special initial guess for Newton iteration applied to the problem $f(t)=0$ in case (b) is provided using the solution of the Riccati differential equation with parameters $c_{0}=c_{1}=0$ when $\zeta \mathrm{a}>0$ :

$$
t_{\text {guess }}= \begin{cases}\frac{2 \zeta}{v_{\circ}+\sqrt{4 \zeta \mathrm{a}+v_{\circ}^{2}}} & \zeta \mathrm{a}>0 \\ 0 & \text { otherwise. }\end{cases}
$$

Because $f(t)$ is convex, $f\left(t_{\text {guess }}\right) \geq 0$ and using Corollary 13 the convergence is monotone. In the cases $(c)(d)$ and $(e)$ a direct application of the Newton method with $t_{\text {guess }}=0$ can cause the iterations to exit the interval of definition of the function, $\left(t_{\min }, t_{\max }\right)$. To avoid this dangerous behaviour, without using a damped Newton method, we construct the iterations as follows. From the fact that $\lim _{t \rightarrow t_{\min }} f(t)=-\infty$, we can approximate the function $f(t)$ with a sequence of model functions $g_{k}(t)$ :

$$
g_{k}(t)=a_{k}-\frac{b_{k}}{t-t_{\min }}, \quad a_{k}=f^{\prime}\left(t_{k}\right)\left(t_{k}-t_{\min }\right)+f\left(t_{k}\right), \quad b_{k}=f^{\prime}\left(t_{k}\right)\left(t_{k}-t_{\min }\right)^{2}
$$

where $a_{k}$ and $b_{k}$ were computed imposing $g_{k}\left(t_{k}\right)=f\left(t_{k}\right)$ and $g_{k}^{\prime}\left(t_{k}\right)=f^{\prime}\left(t_{k}\right)$. The step $t_{k+1}$ is obtained solving $g_{k}\left(t_{k+1}\right)=0$ :

$$
t_{k+1}=t_{\min }+\frac{b_{k}}{a_{k}}=t_{\min }+\frac{f^{\prime}\left(t_{k}\right)\left(t_{k}-t_{\min }\right)^{2}}{f^{\prime}\left(t_{k}\right)\left(t_{k}-t_{\min }\right)+f\left(t_{k}\right)}=t_{k}-\frac{f\left(t_{k}\right)}{f^{\prime}\left(t_{k}\right)+\frac{f\left(t_{k}\right)}{t_{k}-t_{\min }}}
$$

From this construction, if $f\left(t_{k}\right)>0$ and $t_{k}>t_{\min }$ then $t_{\min }<t_{k+1}<t_{k}$, if $f\left(t_{k}\right)<0$, then we are in the hypotheses of Corollary 13 and we can safely employ Newton's method that gives monotone convergence. In conclusion we model the iterations as stated in Algorithm 4.

Lemma 14 (c-d-e). In the cases $(c)-(d)-(e)$ Algorithm 4 converges to the solution $f\left(t_{\star}\right)=0$ with quadratic convergence.

Proof. If $f\left(t_{k_{0}}\right)<0$ for a certain $k_{0}$, then the hypotheses of Corollary 13 are respected, hence the quadratic monotone convergence follows from the convergence of Newton's method. Otherwise, let $t_{\star}$ be the root of $f\left(t_{\star}\right)$, because the steps are negative, we have a monotone decreasing sequence bounded from below, which admits the limit $\bar{t}$. This limit cannot be $\bar{t}>t_{\star}$ because $f(\bar{t})>0$, hence the step remains different from zero. Therefore let $\varepsilon_{k}=t_{k}-t_{\star}$, then we have

$$
\begin{aligned}
\varepsilon_{k+1} & =\varepsilon_{k}-\frac{f\left(t_{k}\right)-f\left(t_{\star}\right)}{f^{\prime}\left(t_{k}\right)+\delta_{k}^{+}\left(f\left(t_{k}\right)-f\left(t_{\star}\right)\right) /\left(t_{k}-t_{\min }\right)} \\
& =\varepsilon_{k}-\frac{f\left(t_{\star}\right)+\varepsilon_{k} f^{\prime}\left(t_{k}\right)+\frac{1}{2} \varepsilon_{k}^{2} f^{\prime \prime}\left(\omega_{k}\right)-f\left(t_{\star}\right)}{f^{\prime}\left(t_{k}\right)+\varepsilon_{k} \delta_{k}^{+} f^{\prime}\left(\zeta_{k}\right) /\left(t_{k}-t_{\min }\right)} \\
& =\frac{1}{2} \varepsilon_{k}^{2} \frac{f^{\prime \prime}\left(\omega_{k}\right)\left(t_{k}-t_{\min }\right)+2 \delta_{k}^{+} f^{\prime}\left(\zeta_{k}\right)}{f^{\prime}\left(t_{k}\right)\left(t_{k}-t_{\min }\right)+\varepsilon_{k} \delta_{k}^{+} f^{\prime}\left(\zeta_{k}\right)},
\end{aligned}
$$

that is,

$$
\lim _{k \rightarrow \infty} \frac{\varepsilon_{k+1}}{\varepsilon_{k}^{2}}=\frac{1}{2} \frac{f^{\prime \prime}\left(t_{\star}\right)\left(t_{\star}-t_{\min }\right)+2 \delta_{k}^{+} f^{\prime}\left(t_{\star}\right)}{f^{\prime}\left(t_{\star}\right)\left(t_{\star}-t_{\min }\right)}=\frac{1}{2} \frac{f^{\prime \prime}\left(t_{\star}\right)}{f^{\prime}\left(t_{\star}\right)}+\frac{\delta}{t_{\star}-t_{\min }}=C,
$$

Hence the convergence is quadratic with constant $C$, which is bigger if the covergence is from the right side. 
Remark 3. It is also possible to use higher order Newton methods, for example, for order 3 or 4 see [14]. The second derivative $f^{\prime \prime}$ is available analytically, thus the Halley method can be applied too [28] (or [32] for a survey of one dimensional methods), for higher order methods see for example [2, 25].

\section{The Optimal Control Problem in the curvilinear abscissa $s$}

With the change of coordinates from time $t$ to space $s$ as independent variable, it is possible to reformulate the OCP (1), passing from free time to a fixed domain. The change of variable is possible in practice using the results of the Section 5. Let $\tilde{v}(s)=v(t(s))$ be the transformed function of the velocity parametrised as a function of $s$ as showed in Corollary 7 , then the minimum time optimal control problem (1) can be reformulated as finding $a(s) \in\left[-\mathrm{a}_{\text {brake }}\right.$, $\left.\mathrm{a}_{\text {push }}\right]$ that minimises

$$
\begin{aligned}
\text { Minimise } \quad T & =\int_{0}^{L} \frac{\mathrm{d} s}{|\tilde{v}(s)|} \\
\text { subject to: } \quad \tilde{v}^{\prime}(s) & =\frac{\mathrm{a}(s)}{\tilde{v}(s)}-c_{0}-c_{1} \tilde{v}(s), \quad \tilde{v}(0)=v_{i}, \quad \tilde{v}(L)=v_{f}, \quad-\mathrm{a}_{\text {brake }} \leq \mathrm{a}(s) \leq \mathrm{a}_{\text {push }},
\end{aligned}
$$

The complete solution of the Optimal Control Problem (19) is reduced to finding the optimal switching point $s_{\sigma}$ such that the solution is written as

$$
\tilde{v}(s)= \begin{cases}\tilde{v}_{L}(s) & s<s_{\sigma} \\ \tilde{v}_{R}(s) & s \geq s_{\sigma},\end{cases}
$$

where $\tilde{v}_{L}(s)$ and $\tilde{v}_{R}(s)$ are the solutions of the ODE

$$
\begin{array}{ll}
\tilde{v}_{L}^{\prime}(s)=\frac{\mathrm{a}_{\text {push }}}{\tilde{v}_{L}(s)}-c_{0}-c_{1} \tilde{v}_{L}(s), & \tilde{v}_{L}(0)=v_{i}, \\
\tilde{v}_{R}^{\prime}(s)=-\frac{\mathrm{a}_{\mathrm{brake}}}{\tilde{v}_{R}(s)}-c_{0}-c_{1} \tilde{v}_{R}(s), & \tilde{v}_{R}(L)=v_{f} .
\end{array}
$$

Remark 4. Indeed we notice that while it is possible to solve the dynamic system of (1) as explicit functions of $v(t)$ and $s(t)$, the differential equation (19) for $\tilde{v}(s)$ admits only an implicit solution that is impractical to subsolve with respect to $s$. Therefore we employ the knowledge of the analytic solutions presented in the previous sections for $v(t)$ and $s(t)$ together with the numeric change of variable of Section 5.

The computation of the switching point $s_{\sigma}$ is done equating the arcs of positive acceleration with the corresponding arcs of negative acceleration. It results a single nonlinear equation $g\left(s_{\sigma}\right)=0$ where:

$$
g(s)=\tilde{v}_{L}(s)-\tilde{v}_{R}(s)
$$

To solve $g\left(s_{\sigma}\right)=0$ we simply use the Newton method: the derivative of $g(s)$ is readily given by

$$
g^{\prime}(s)=\tilde{v}_{L}^{\prime}(s)-\tilde{v}_{R}^{\prime}(s)=\frac{\mathrm{a}_{\text {push }}}{\tilde{v}_{L}(s)}+\frac{\mathrm{a}_{\mathrm{brake}}}{\tilde{v}_{R}(s)}+c_{1}\left(\tilde{v}_{R}(s)-\tilde{v}_{L}(s)\right)
$$

and the latter is deduced from (20).

\section{Numerical Tests}

We present herein four numerical tests for various choices of the parameters of the problem, namely we consider the parameters $c_{0}, c_{1}, \mathrm{a}_{\text {push }}$ and $\mathrm{a}_{\text {brake. }}$. The tests are done with the stable formulas for velocity and space of Section 4 with the change of variable from $t$ to $s$ explained in Section 5. 
Table 2: Table of fixed and varying parameters

Fixed Parameters

\begin{tabular}{c|llllllllll}
\hline$v(0)[\mathrm{m} / \mathrm{s}]$ & $v(L)[\mathrm{m} / \mathrm{s}]$ & $L[\mathrm{~m}]$ & $\mathrm{a}_{\text {push }}\left[\mathrm{m} / \mathrm{s}^{2}\right]$ & $\mathrm{a}_{\text {brake }}\left[\mathrm{m} / \mathrm{s}^{2}\right]$ & $c_{0}[1 / \mathrm{m}]$ & $c_{1}[1 / \mathrm{s}]$ \\
\hline 6 & 5 & 100 & 2 & \multicolumn{10}{c}{2} & & 0.01 & 0.01 \\
\hline \multicolumn{8}{c}{ Varying Parameters } \\
\hline$c_{0}[1 / \mathrm{m}]$ & 0 & $10^{-5}$ & 0.01 & 0.05 & 0.1 & 0.2 & 0.3 & 0.4 & 0.5 \\
\hline$c_{1}[1 / \mathrm{s}]$ & 0 & 0.005 & 0.01 & 0.02 & 0.03 & & & & \\
\hline $\mathrm{a}_{\text {push }}\left[\mathrm{m} / \mathrm{s}^{2}\right]$ & $10^{-6}$ & 0.01 & 0.05 & 0.1 & 0.25 & 1 & 2 & 10 & \\
\hline $\mathrm{a}_{\text {brake }}\left[\mathrm{m} / \mathrm{s}^{2}\right]$ & $10^{-6}$ & 0.01 & 0.05 & 0.1 & 0.25 & 1 & 2 & 10 & \\
\hline
\end{tabular}

It is worth noticing and pointing out that although the solutions of the Riccati differential equation derived in Section 3 are analytic, a direct naïve implementation of such equations leads to numerical instabilities, which is the main motivation that led to this study. In particular, things go wrong when the friction parameters $c_{0}$ and $c_{1}$ are near to zero, producing a large error in the division by (near) zero coefficients, or, even if they are far from being zero, but $w$ results zero or almost zero, for example when the acceleration is negative (braking phase) and $c_{0}^{2}-4 \mathrm{a} c_{1} \approx 0$. The instabilities are also evident in the computation of the time and space domains of the equations.

In the first test we fix the initial/final positions with their speeds and we compute the time optimal control problem with different values of the friction coefficient $c_{0}$. The fixed parameters and the coefficients for the aerodynamic drag are resumed in Table 2. The result is showed in Figure 3.1, the curves are ordered from top to bottom according to the increasing value of $c_{0}$ : the top most curve is for $c_{0}=0$ and the last curve on the bottom is the one for $c_{0}=0.5$. Starting from above, the first five curves represent a typical situation of a positive acceleration that produces an increment in the velocity, followed by a braking phase. The flat zones indicate that the asymptotic speed $v_{\infty}$ has been reached. The sixth curve shows a case where the friction is too intense and a positive acceleration produces a reduction of the speed that settles at $v_{\infty}$; the final condition is then met by a rapid braking phase. The last two curves (dashed lines) at the bottom of Figure 3.1 show two infeasible manoeuvres, which means the friction is too high (or equivalently the acceleration $\mathrm{a}_{\text {push }}$ is too weak) to reach the final point. Also here the flat zones indicate the asymptotic velocity $v_{\infty}$. The second example shows the behaviour of changing the aerodynamic coefficient $c_{1}$. We keep the parameters previously fixed in Table 2 and consider the values for $c_{1}$ from $c_{1}=0$ to $c_{1}=0.03$ as in Table 2 . The result is showed in Figure 3.2, where the curves are ordered from top to bottom according to increasing values of $c_{1}$, e.g. the higher the friction, the slower the velocity.

The third experiment makes the value of $\mathrm{a}_{\text {push }}$ change from $\mathrm{a}_{\text {push }}=10^{-6}$ to $\mathrm{a}_{\text {push }}=10$. The other values are kept as in the previous tests. The curves of Figure 3.3 are ordered from top to bottom according to the decreasing values of $\mathrm{a}_{\text {push }}$. The first four are admissible manoeuvres that share a common deceleration curve, the last four curves (dashed) are non admissible solutions because the acceleration $\mathrm{a}_{\text {push }}$ is not strong enough to reach the final position.

The fourth test makes the value of $a_{\text {brake }}$ change from $a_{\text {brake }}=10^{-6}$ to $a_{\text {brake }}=10$. The curves of Figure 3.4 are ordered from top to bottom according to the increasing values of $a_{\text {brake }}$, that is, the stronger is the braking, the shorter is the braking phase. The curves are admissible manoeuvres that share a common acceleration curve and meet in the final point.

\section{Conclusions, Future Work and Applications}

In this paper we developed an accurate and fast numerical algorithm for the semi-analytic solution for the time optimal control of a car-like model from an initial to a final position and velocity. The novelty of the OCP is the presence of the quadratic term in the ODE for the velocity, which yields a nonlinear differential equation of Riccati kind. The nature of the OCP being Bang-Bang allows the analytic solution of the ODEs and thus the reduction of the problem to the numeric solution of a curve intersection. An immediate consequence is the avoidance of the complete numeric integration of the dynamic system and hence a very low computational time. A rough estimate 

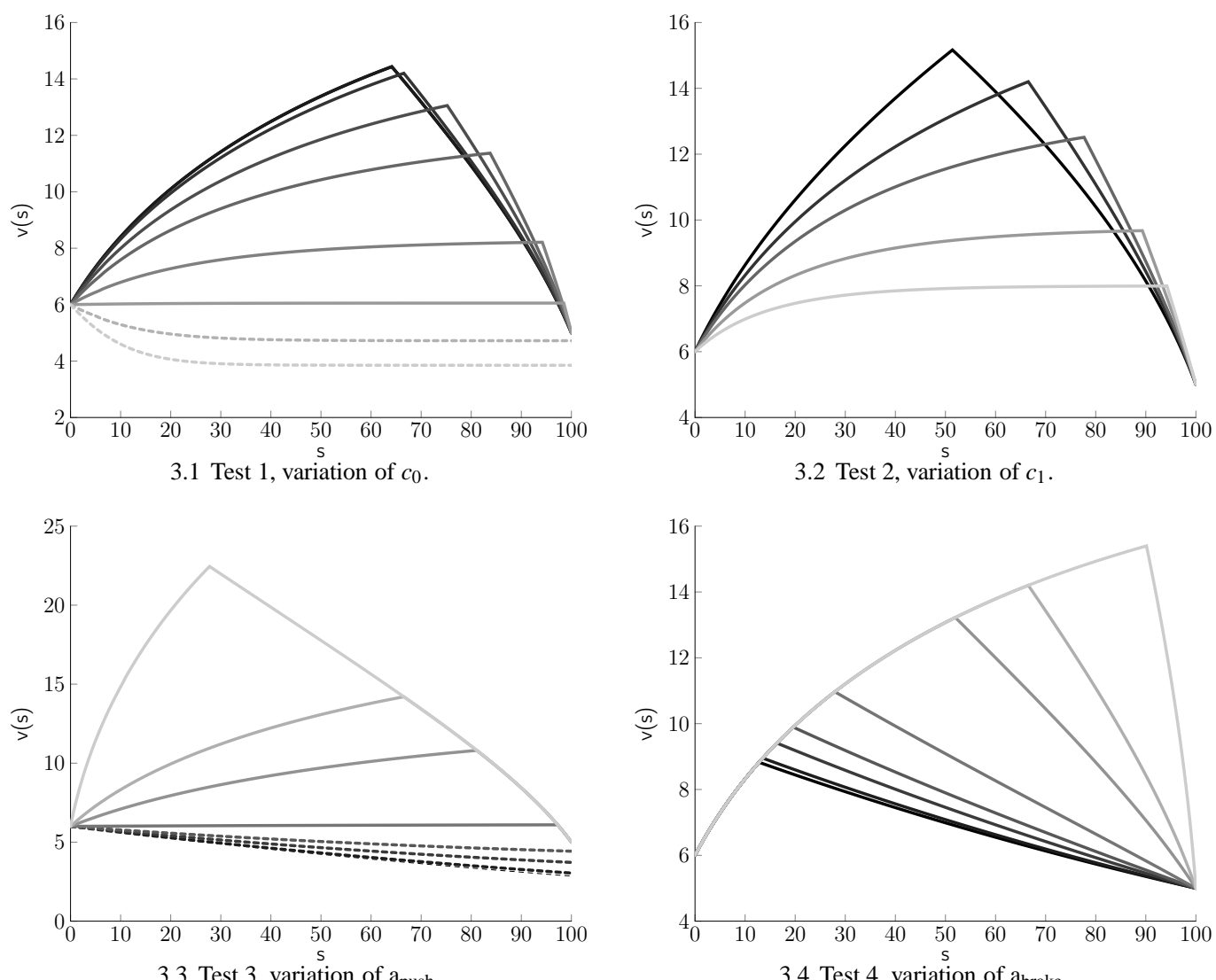

Figure 3: The four pictures show the graphical result of the numerical tests. Solid lines are feasible manoeuvres while dashed lines are unfeasible manoeuvres. Parameters of the computed solutions are taken from Table 2

of the computational cost for the present semi-analytic solution of the OCP is around 1 microsecond with a $\mathrm{C}++$ implementation, against a pure numerical solution with Pins or GPOPS-II of around 1 second.

We successfully used it in the solution of the more general optimal control problem of steering a car-like model with the additional constraint on the maximum lateral acceleration and a given trajectory expressed as clothoid curve, see [17]. The effectiveness of the algorithm presented in this paper was mandatory in the construction of the tool, because during the optimisation process, many instances of the Bang-Bang algorithm were required, most of them with parameters that came from other computational processes, yielding non standard situations. The naïve implementation of the analytic solution was found to be insufficient for that purpose, therefore we developed the stable formulas.

As a future work based on the present building block, we want to solve the optimal control herein presented with the limitation on the lateral acceleration and with a trajectory given by fixed sequence of clothoids, not just one. Another future study is the design of a tool that gives the time optimal trajectory on a road, in terms of clothoids, combining the present module with a planning algorithm.

\section{Acknowledgements}

The research activity described in this paper has received funding from the University of Trento within the project "OptHySYS - Optimisation techniques for Hybrid dynamical SYStems: from theory to applications". 


\section{Appendix: Algorithms collected}
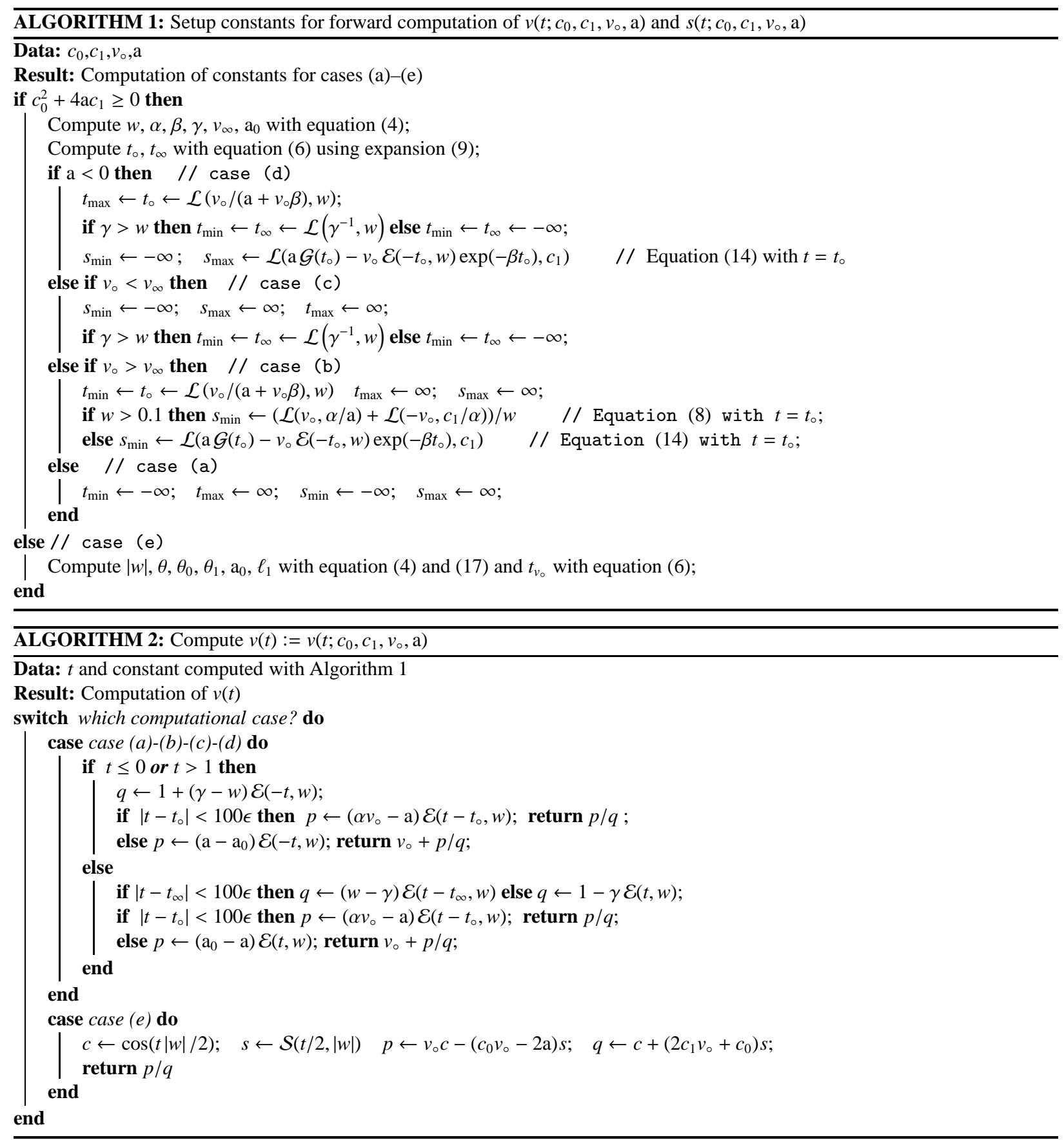


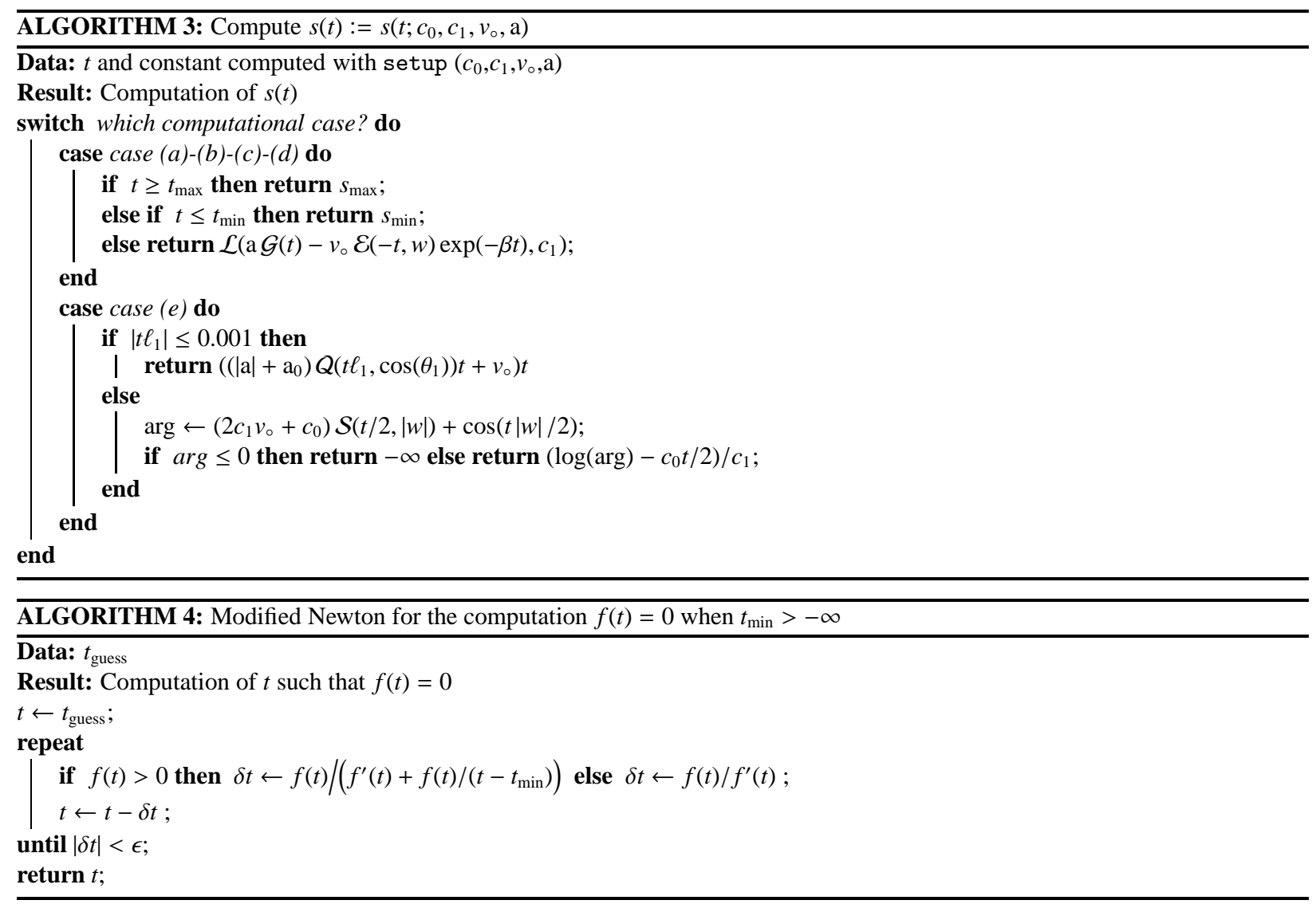

\section{References}

[1] Abramowitz, M., Stegun, I.: Handbook of Mathematical Functions with Formulas, Graphs, and Mathematical Tables. No. 55 in National Bureau of Standards Applied Mathematics Series. U.S. Government Printing Office, Washington, D.C. (1964)

[2] Amat, S., Hernández, M., Romero, N.: A modified chebyshev's iterative method with at least sixth order of convergence. Applied Mathematics and Computation 206(1), 164 - 174 (2008). DOI http://dx.doi.org/10.1016/j.amc.2008.08.050. URL http://www . sciencedirect . com/science/article/pii/S0096300308006449

[3] Amditis, A., Bertolazzi, E., Bimpas, M., Biral, F., Bosetti, P., Da Lio, M., Danielsson, L., Gallione, A., Lind, H., Saroldi, A., Sjögren, A.: A holistic approach to the integration of safety applications: The insafes subproject within the european framework programme 6 integrating project prevent. IEEE Transactions on Intelligent Transportation Systems 11(3), 554-566 (2010). DOI 10.1109/TITS.2009.2036736

[4] Ascher, U., Mattheij, R., Russell, R.: Numerical Solution of Boundary Value Problems for Ordinary Differential Equations. Society for Industrial and Applied Mathematics (1995). DOI 10.1137/1.9781611971231

[5] Athans, M., Falb, P.: Optimal Control: An Introduction to the Theory and Its Applications. Lincoln Laboratory publications. McGraw-Hill (1966)

[6] Bertolazzi, E., Biral, F., Da Lio, M.: Symbolic-numeric efficient solution of optimal control problems for multibody systems. Journal of Computational and Applied Mathematics 185(2), 404-421 (2006). DOI 10.1016/j.cam.2005.03.019

[7] Bertolazzi, E., Biral, F., Da Lio, M., Saroldi, A., Tango, F.: Supporting drivers in keeping safe speed and safe distance: The saspence subproject within the european framework programme 6 integrating project prevent. IEEE Transactions on Intelligent Transportation Systems 11(3), 525-538 (2010). DOI 10.1109/TITS.2009.2035925

[8] Bertolazzi, E., Frego, M.: G1 fitting with clothoids. Mathematical Methods in the Applied Sciences 38(5), 881-897 (2015). DOI 10.1002/ mma.3114

[9] Betts, J.: Practical Methods for Optimal Control and Estimation Using Nonlinear Programming, second edn. Society for Industrial and Applied Mathematics (2010). DOI 10.1137/1.9780898718577

[10] Betts, J.T., Campbell, S.L., Engelsone, A.: Direct transcription solution of optimal control problems with higher order state constraints: theory vs practice. Optimization and Engineering 8(1), 1-19 (2007). DOI 10.1007/s11081-007-9000-8

[11] Biral, F., Bertolazzi, E., Da Lio, M.: The Optimal Manoeuvre, chap. 5, pp. 119-154. John Wiley \& Sons, Ltd (2014). DOI 10.1002/ 9781118536391.ch5

[12] Büskens, C., Wassel, D.: The ESA NLP solver WORHP. In: G. Fasano, J.D. Pintér (eds.) Modeling and Optimization in Space Engineering, Springer Optimization and Its Applications, vol. 73, pp. 85-110. Springer New York (2013). DOI 10.1007/978-1-4614-4469-5_4 
[13] Campbell, S.L., Betts, J.T.: Comments on direct transcription solution of dae constrained optimal control problems with two discretization approaches. Numerical Algorithms pp. 1-32 (2016). DOI 10.1007/s11075-016-0119-6

[14] Chun, C., Neta, B.: Certain improvements of newton's method with fourth-order convergence. Applied Mathematics and Computation 215(2), 821 - 828 (2009). DOI 10.1016/j.amc.2009.06.007

[15] Conte, S.D., Boor, C.W.D.: Elementary Numerical Analysis: An Algorithmic Approach, 3rd edn. McGraw-Hill Higher Education (1980)

[16] Dubins, L.E.: On curves of minimal length with a constraint on average curvature, and with prescribed initial and terminal positions and tangents. American Journal of mathematics pp. 497-516 (1957)

[17] Frego, M., Bertolazzi, E., Biral, F., Fontanelli, D., Palopoli, L.: Semi-analytical minimum time solutions for a vehicle following clothoidbased trajectory subject to velocity constraints. In: European Control Conference (ECC), p. 7 (2016)

[18] Frego, M., Bertolazzi, E., Biral, F., Fontanelli, D., Palopoli, L.: Semi-Analytical Minimum Time Solutions with Velocity Constrainst for Trajectory Following of Vehicles. Automatica (2016). Submitted

[19] Frego, M., Bevilacqua, P., Bertolazzi, E., Biral, F., Fontanelli, D., Palopoli, L.: Trajectory Planning for car-like vehicles: a modular approach. Conference on Decision and Control (CDC 2016) (2016). Submitted

[20] Gerdts, M., Xausa, I.: Avoidance trajectories using reachable sets and parametric sensitivity analysis. IFIP Advances in Information and Communication Technology 391 AICT, 491-500 (2013). DOI 10.1007/978-3-642-36062-6_49

[21] Houska, B., Ferreau, H., Diehl, M.: ACADO Toolkit - An Open Source Framework for Automatic Control and Dynamic Optimization. Optimal Control Applications and Methods 32(3), 298-312 (2011)

[22] Landry, C., Gerdts, M., Henrion, R., Hömberg, D.: Path-planning with collision avoidance in automotive industry. IFIP Advances in Information and Communication Technology 391 AICT, 102-111 (2013). DOI 10.1007/978-3-642-36062-6_11

[23] Lio, M.D., Biral, F., Bertolazzi, E., Galvani, M., Bosetti, P., Windridge, D., Saroldi, A., Tango, F.: Artificial co-drivers as a universal enabling technology for future intelligent vehicles and transportation systems. IEEE Transactions on Intelligent Transportation Systems 16(1), 244-263 (2015). DOI 10.1109/TITS.2014.2330199

[24] Lipp, T., Boyd, S.: Minimum-time speed optimisation over a fixed path. International Journal of Control 87(6), 1297-1311 (2014). DOI 10.1080/00207179.2013.875224

[25] Lotfi, T., Sharifi, S., Salimi, M., Siegmund, S.: A new class of three-point methods with optimal convergence order eight and its dynamics. Numerical Algorithms 68(2), 261-288 (2014). DOI 10.1007/s11075-014-9843-y

[26] Mazzia, F., Sgura, I.: Numerical approximation of nonlinear BVPs by means of BVMs. Applied Numerical Mathematics 42(1-3), 337 - 352 (2002). DOI http://dx.doi.org/10.1016/S0168-9274(01)00159-3

[27] Mazzia, F., Trigiante, D.: Numerical solution of optimal control problems. In: S. Sivasundaram (ed.) Advances in Dynamics and Control: Theory Methods and Applications, Mathematical problems in engineering, aerospace and sciences. Cambridge Scientific Publ. (2011)

[28] Neta, B., Scott, M.: On a family of halley-like methods to find simple roots of nonlinear equations. Applied Mathematics and Computation 219(15), 7940 - 7944 (2013). DOI 10.1016/j.amc.2013.02.035

[29] Nocedal, J., Wright, S.: Numerical Optimization. Springer Series in Operations Research and Financial Engineering. Springer New York (2006)

[30] Olver, F.J., Lozier, D., Boisvert, R., Clark, C.: NIST handbook of mathematical functions. U.S. Department of Commerce National Institute of Standards and Technology, Washington, DC (2010). With CD-ROM

[31] Patterson, M.A., Rao, A.V.: GPOPS-II: A MATLAB software for solving multiple-phase optimal control problems using hp-adaptive gaussian quadrature collocation methods and sparse nonlinear programming. ACM Trans. Math. Softw. 41(1), 1:1-1:37 (2014). DOI 10.1145/2558904

[32] Petković, M.S., Neta, B., Petković, L.D., Džunić, J.: Multipoint methods for solving nonlinear equations: A survey. Applied Mathematics and Computation 226, 635 - 660 (2014). DOI 10.1016/j.amc.2013.10.072

[33] Rao, A.: Trajectory optimization: A survey. Lecture Notes in Control and Information Sciences 455 LNCIS, 3-21 (2014). DOI 10.1007/ 978-3-319-05371-4_1

[34] Rizano, T., Fontanelli, D., Palopoli, L., Pallottino, L., Salaris, P.: Global path planning for competitive robotic cars. In: Decision and Control (CDC), 2013 IEEE 52nd Annual Conference on, pp. 4510-4516. IEEE (2013)

[35] Sanfelice, R., Yong, S., Frazzoli, E.: On minimum-time paths of bounded curvature with position-dependent constraints. Automatica 50(2), 537-546 (2014). URL http://ares.lids.mit.edu/papers/Sanfelice.Yong.ea.AUT13.pdf

[36] Velenis, E., Tsiotras, P.: Optimal velocity profile generation for given acceleration limits: theoretical analysis. In: American Control Conference, 2005. Proceedings of the 2005, pp. 1478-1483 vol. 2 (2005). DOI 10.1109/ACC.2005.1470174

[37] Velenis, E., Tsiotras, P.: Minimum-time travel for a vehicle with acceleration limits: Theoretical analysis and receding-horizon implementation. Journal of Optimization Theory and Applications 138(2), 275-296 (2008). DOI 10.1007/s10957-008-9381-7

[38] Verscheure, D., Demeulenaere, B., Swevers, J., Schutter, J.D., Diehl, M.: Time-optimal path tracking for robots: A convex optimization approach. IEEE Transactions on Automatic Control 54(10), 2318-2327 (2009). DOI 10.1109/TAC.2009.2028959

[39] Wächter, A., Biegler, T.L.: On the implementation of an interior-point filter line-search algorithm for large-scale nonlinear programming. Mathematical Programming 106(1), 25-57 (2005). DOI 10.1007/s10107-004-0559-y

[40] Wyk, E.J.V., Falugi, P., Kerrigan, E.: ICLOCS (2010). URL http://www.ee.ic.ac.uk/ICLOCS

[41] Zaitsev, V., Polyanin, A.: Handbook of Exact Solutions for Ordinary Differential Equations. CRC Press (2002) 